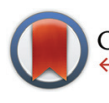

CrossMark

Cite this: Polym. Chem., 2016, 7, 851

\title{
Addition of water to an alcoholic RAFT PISA formulation leads to faster kinetics but limits the evolution of copolymer morphology
}

\author{
E. R. Jones, ${ }^{a}$ M. Semsarilar, ${ }^{a}$ P. Wyman, ${ }^{b}$ M. Boerakker ${ }^{b}$ and S. P. Armes ${ }^{{ }^{a}}$
}

RAFT dispersion polymerization of benzyl methacrylate (BzMA) has been used previously (E. R. Jones, et al., Macromolecules, 2012, 45, 5091) to prepare poly(2-(dimethylamino)ethyl methacrylate)-poly(benzyl methacrylate) (PDMA-PBzMA) diblock copolymer nanoparticles in ethanol via polymerizationinduced self-assembly (PISA). However, the rate of polymerization was relatively slow, with incomplete monomer conversions being obtained when targeting higher mean degrees of polymerization (DP) even after $24 \mathrm{~h}$ at $70{ }^{\circ} \mathrm{C}$. Herein we examine the effect of the addition of up to $20 \% \mathrm{w} / \mathrm{w}$ water co-solvent on the kinetics of BzMA polymerization for this PISA formulation. Significantly faster polymerizations were observed: for a target DP of 200,90\% BzMA conversion was achieved within just $6 \mathrm{~h}$ in the presence of $20 \% \mathrm{w} / \mathrm{w}$ water, compared to only $35 \%$ conversion in anhydrous ethanol under the same conditions. This rate enhancement enables much higher mean DPs to be obtained for the core-forming PBzMA and is attributed to greater partitioning of the BzMA monomer within the particles, which increases the local monomer concentration. However, the presence of water adversely affected the evolution of copolymer morphology from spheres to worms to vesicles when employing a relatively short PDMA chain transfer agent, with only kinetically-trapped spheres being obtained at higher levels of added water. Aqueous electrophoresis studies indicate that the PDMA stabilizer chains acquired partial cationic charge in the presence of water. This leads to more efficient inter-particle repulsion, thus preventing the sphere-sphere fusion events required for an evolution in morphology. In summary, the addition of water to such PISA formulations allows the more efficient synthesis of spherical nanoparticles, but should be used with caution if either diblock copolymer worms or vesicles are desired.

Received 9th November 2015 Accepted 25th November 2015 DOI: $10.1039 / c 5 p y 01795 \mathrm{e}$

www.rsc.org/polymers solvent: the resulting amphiphilic diblock copolymer undergoes spontaneous in situ self-assembly to form diblock copolymer nano-objects. PISA formulations are robust, efficient and highly versatile: aqueous emulsion, aqueous dispersion and non-aqueous polymerization protocols have been developed using various LRP chemistries. ${ }^{5-19}$

Over the past few years, there have been many literature reports of RAFT dispersion polymerization formulations being conducted in alcoholic media. ${ }^{19-27}$ Of particular relevance to the present study, we reported an all-methacrylic RAFT dispersion polymerization protocol based on chain extension of a poly(2-(dimethylamino)ethyl methacrylate) (PDMA) macro-CTA using benzyl methacrylate (BzMA) in ethanol. ${ }^{14}$ Substantially higher final monomer conversions were obtained compared to those previously reported for similar PISA formulations based on the polymerization of styrene..$^{20,22,28}$ The RAFT dispersion polymerization of BzMA displayed relatively good pseudoliving character, as judged by GPC analysis. When using a relatively short PDMA stabilizer block with a mean degree of polymerization (DP) of 31, either spheres, worms or vesicles 
could be produced when targeting longer core-forming PBzMA blocks. Very recently, this formulation was revisited to examine the mechanism of particle growth during this PISA synthesis. ${ }^{29}$ As expected, using a longer PDMA macro-CTA (DP = 94) limited the morphology of the resulting particles to kineticallytrapped spheres. A monotonic increase in particle diameter was observed when targeting progressively higher DPs for the core-forming block. Small angle X-ray scattering (SAXS) and multi-angle laser light scattering (MALLS) studies confirmed a linear relationship between micelle aggregation number and PBzMA DP, which suggests that particle growth during such PISA syntheses occurs either by exchange of copolymer chains between micelles, and/or via sphere-sphere fusion events. However, the relatively slow rate of BzMA polymerization led to substantially incomplete monomer conversions when targeting PBzMA DPs greater than 700, which placed an upper limit on the mean spherical particle diameter that could be obtained.

Several groups have utilized alcohol/water mixtures for similar RAFT alcoholic dispersion polymerization formulations. ${ }^{30-33}$ For example, Charleux and co-workers investigated both dispersion and emulsion polymerization formulations for the chain extension of a poly(methacrylic acid-copoly(ethylene oxide) monomethyl ether methacrylate) (P(MAAPEOMA)) macro-CTA with BzMA in ethanol/water or 1,4dioxane/water solvent mixtures. ${ }^{30}$ it was found that increasing the proportion of water co-solvent significantly increased the rate of BzMA polymerization when using a fixed [BzMA $]_{0} /$ [macro-CTA] molar ratio of 300. Importantly, GPC analyses indicated fairly well-controlled polymerizations in all cases. Moreover, targeting the same diblock composition produced various copolymer morphologies depending on the precise solvent composition. Although changes in copolymer morphology were noted in this study, the effect of varying the diblock copolymer composition was not systematically investigated and only limited kinetic data were presented.

Zhang and co-workers also examined various ethanol/water mixtures for the RAFT dispersion polymerization of styrene. They investigated both the homopolymerization of styrene in the presence of a PNVP stabilizer and the synthesis of polystyrene-based block copolymer nanoparticles. ${ }^{31-33}$ When using the PNVP stabilizer, styrene was polymerized in the presence of an $S, S^{\prime}$-bis $\left(\alpha, \alpha^{\prime}\right.$-dimethyl- $\alpha^{\prime \prime}$-acetic acid) trithiocarbonate (BDMAT) RAFT CTA. ${ }^{32}$ Kinetic data were obtained for styrene polymerizations containing $0-30 \% \mathrm{w} / \mathrm{w}$ water. As reported for $\mathrm{BzMA}^{30}{ }^{30}$ the rate of styrene polymerization increased significantly as the water content was increased. This observation was discussed in some detail, with close reference to a prior study on the TEMPO-mediated polymerization of styrene. ${ }^{34}$ Zhang et al. proposed that, prior to micellar nucleation, a relatively slow homogeneous solution polymerization occurred. An enhanced rate of polymerization was observed immediately after the onset of nucleation, since polymerization now took place mainly within monomer-swollen micelles. Essentially the same two-stage kinetic model was previously reported by
Blanazs and co-workers for the RAFT aqueous dispersion polymerization of HPMA. $^{35}$

Based on the PISA literature, it is clear that the precise solvent composition can have a strong influence on both the polymerization kinetics and final copolymer morphology observed for RAFT dispersion polymerization formulations. In the present study, the effect of addition of $0-20 \% \mathrm{w} / \mathrm{w}$ water co-solvent to the ethanolic RAFT dispersion polymerization of BzMA is examined in detail. ${ }^{14}$ Since water is a poor solvent for BzMA, its presence should promote stronger monomer partitioning within the micelles formed during nucleation. This should result in a higher local BzMA concentration within the micelles, with a concomitant rate enhancement. Thus, in principle, this should enable access to higher PBzMA DPs and hence larger particle diameters. The extent to which this approach limits the subsequent evolution of the copolymer morphology is also explored.

\section{Experimental}

\section{Materials}

All reagents were purchased from Sigma-Aldrich (UK) and were used as received unless otherwise noted. Either 4,4'-azobis(4cyanovaleric acid) (ACVA)) or 2,2'-azobisisobutyronitrile (AIBN) was used as an initiator. Benzyl methacrylate (96\%) was passed through an inhibitor removal column (Sigma) prior to use. $\mathrm{CDCl}_{3}, \mathrm{CD}_{2} \mathrm{Cl}_{2}$ and $\mathrm{C}_{2} \mathrm{D}_{5} \mathrm{OD}$ were purchased from Goss Scientific (Nantwich, UK). Anhydrous ethanol ( $\geq 99.5 \%)$ was used to ensure the precise water content of solvent mixtures was known. Deionized water was used in polymerizations conducted in solvent mixtures and was degassed prior to addition. 4-Cyano-4-(2-phenyl-ethanesulfanylthiocarbonyl) sulfanylpentanoic acid (PETTC) was prepared in-house as reported previously. ${ }^{14}$

\section{Synthesis of poly(2-(dimethylamino)ethyl methacrylate) (PDMA) macro-CTA}

A round-bottomed flask was charged with 2-(dimethylamino)ethyl methacrylate (DMA; $40.0 \mathrm{~g}, 254 \mathrm{mmol})$, PETTC (2.160 g, $6.36 \mathrm{mmol}$; target DP = 40), ACVA $(178 \mathrm{mg}, 0.64 \mathrm{mmol}$; PETTC/ ACVA $=10)$ and THF $(40.0 \mathrm{~g})($ target DP $=40)$. The sealed reaction vessel was purged with nitrogen and placed in a preheated oil bath at $70{ }^{\circ} \mathrm{C}$ for $8.5 \mathrm{~h}$. The resulting polymer (monomer conversion $=87 \% ; M_{\mathrm{n}}=6700 \mathrm{~g} \mathrm{~mol}^{-1}, M_{\mathrm{w}} / M_{\mathrm{n}}=$ 1.14) was purified by precipitation into excess petroleum ether. The mean degree of polymerization (DP) of this PDMA macroCTA was calculated to be 43 using ${ }^{1} \mathrm{H}$ NMR spectroscopy by comparing the integrated signals corresponding to the aromatic protons at $7.2-7.4 \mathrm{ppm}$ with those due to the methacrylic polymer backbone at $0.4-2.5$ ppm. A similar protocol utilized [DMA]/[PETTC] ratio of 90 to synthesize a PDMA $_{74}$ macro-CTA $\left(M_{\mathrm{n}}=11300 \mathrm{~g} \mathrm{~mol}^{-1}, M_{\mathrm{w}} / M_{\mathrm{n}}=1.19\right)$. 
Synthesis of poly(2-(dimethylamino)ethyl methacrylate)-poly (benzyl methacrylate) (PDMA-PBzMA) diblock copolymer particles via dispersion polymerization in either ethanol or various ethanol/water mixtures

In a typical RAFT dispersion polymerization synthesis conducted at 15\% w/w solids, BzMA (2.00 g, $11.4 \mathrm{mmol}), \mathrm{PDMA}_{43}$ macro-CTA (402 mg, $0.057 \mathrm{mmol}$ ) and AIBN (1.90 mg, $0.011 \mathrm{mmol}$ ) were dissolved in ethanol $(13.5 \mathrm{~g})$. The reaction mixture was sealed in a round-bottomed flask, purged with nitrogen gas for 20 minutes and then placed in a pre-heated oil bath at $70{ }^{\circ} \mathrm{C}$ for $24 \mathrm{~h}$. The final monomer conversion was determined by ${ }^{1} \mathrm{H}$ NMR analysis in $\mathrm{CDCl}_{3}$ by comparing the integrated PBzMA $\left(\mathrm{CH}_{2}\right)$ signal at $4.9 \mathrm{ppm}$ to that assigned to the two methylene vinyl signals of BzMA monomer at 5.2 and $5.4 \mathrm{ppm}$. In further PDMA-PBzMA diblock copolymer syntheses, the mean DP of the PBzMA block was systematically varied by adjusting the amount of BzMA monomer (target DP 60-2500), and also conducted in various ethanol/water mixtures with water contents ranging between 5 and $20 \% \mathrm{w} / \mathrm{w}$. In reactions using water as a co-solvent, deionized water was purged with nitrogen prior to addition to the reaction solution. Otherwise all other aspects of the experimental protocol were as described for syntheses conducted in anhydrous ethanol.

\section{Copolymer characterization}

Gel permeation chromatography (GPC) was used to assess diblock copolymer molecular weight distributions using an Agilent PL-GPC 50 integrated GPC instrument. The GPC set-up comprised two $5 \mu \mathrm{m}(30 \mathrm{~cm})$ 'Mixed C' columns and a WellChrom K-2301 refractive index detector operating at 950土 $30 \mathrm{~nm}$. THF eluent containing $2.0 \% \mathrm{v} / \mathrm{v}$ triethylamine and $0.05 \% \mathrm{w} / \mathrm{v}$ butylhydroxytoluene (BHT) was used at a flow rate of $1.0 \mathrm{~mL} \mathrm{~min}^{-1}$. A series of ten near-monodisperse linear poly (methyl methacrylate) standards ( $M_{\mathrm{p}}$ ranging from 1280 to $330000 \mathrm{~g} \mathrm{~mol}^{-1}$ ) were purchased from Polymer Laboratories (Church Stretton, UK) and employed for calibration using the above refractive index detector.

${ }^{1} \mathbf{H}$ NMR spectra were acquired using a Bruker $400 \mathrm{MHz}$ spectrometer in either $\mathrm{CDCl}_{3}, \mathrm{CD}_{2} \mathrm{Cl}_{2}, \mathrm{C}_{2} \mathrm{D}_{5} \mathrm{OD}$ or $\mathrm{D}_{2} \mathrm{O}$; all chemical shifts are reported in ppm $(\delta)$ (typically 64 scans were averaged per spectrum).

Dynamic light scattering (DLS) measurements were conducted on highly dilute $(0.10 \% \mathrm{w} / \mathrm{w})$ copolymer dispersions at $20{ }^{\circ} \mathrm{C}$ using a Malvern Instruments Zetasizer Nano series instrument equipped with a $4 \mathrm{~mW}$ He-Ne laser operating at $633 \mathrm{~nm}$. Scattered light was detected at $173^{\circ}$ using an avalanche photodiode detector with high quantum efficiency, and an ALV/LSE-5003 multiple tau digital correlator electronics system. The Stokes-Einstein equation is used to obtain the hydrodynamic diameter; this assumes perfectly monodisperse, non-interacting, spherical particles.

Transmission electron microscopy (TEM) imaging was performed at $100 \mathrm{kV}$ on a Phillips CM100 instrument equipped with a Gatan 1k CCD camera. As-synthesized copolymer dispersions were diluted with ethanol or ethanol/water mixtures at $20{ }^{\circ} \mathrm{C}$ to generate $0.20 \% \mathrm{w} / \mathrm{w}$ dispersions. Copper/palladium TEM grids (Agar Scientific, UK) were surface-coated in-house to yield a thin film of amorphous carbon. The grids were then plasma glow-discharged for 30 seconds to create a hydrophilic surface. Each diblock copolymer dispersion $(0.20 \% \mathrm{w} / \mathrm{w}$, $10 \mu \mathrm{L}$ ) was placed onto a freshly glow-discharged grid for one minute and then blotted with filter paper to remove excess solution. To stain the deposited nanoparticles, a $0.75 \% \mathrm{w} / \mathrm{w}$ aqueous solution of uranyl formate $(10 \mu \mathrm{L})$ was placed on the sample-loaded grid via micropipet for 20 seconds and then carefully blotted to remove excess stain. Each grid was then carefully dried using a vacuum hose.

\section{Results and discussion}

A poly(2-(dimethylamino)ethyl methacrylate) PDMA macro-CTA was synthesized by conventional RAFT solution polymerization in THF, see Scheme 1. The method was as reported previously, ${ }^{14}$ following purification the DP of this PDMA macroCTA was determined to be 43 by ${ }^{1} \mathrm{H}$ NMR. This PDMA 43 macroCTA was then chain-extended with BzMA via RAFT dispersion polymerization, in ethanol or ethanol/water mixtures at $70{ }^{\circ} \mathrm{C}$, using AIBN initiator $([\mathrm{PDMA}] /[\mathrm{AIBN}]=5)$. The kinetics of reactions targeting $\mathrm{PDMA}_{43}-\mathrm{PBzMA}_{200}$ in anhydrous ethanol or ethanol/water mixtures were monitored, see Fig. 1. It is clear that increasing the water content significantly increases the rate of BzMA polymerization. The most dramatic rate enhancement is observed when $20 \% \mathrm{w} / \mathrm{w}$ water is used as a co-solvent. In this case, more than $90 \%$ monomer conversion is attained within $6 \mathrm{~h}$ at $70^{\circ} \mathrm{C}$.

For previous work, in which polymerizations were conducted in pure ethanol, a rate enhancement was observed after an initial induction period. This was attributed to the onset of micellar nucleation at a PBzMA DP of around 50, with the nascent monomer-swollen micelles acting as nanoreactors. ${ }^{14}$ In the present study, polymerizations conducted in various mixed solvents also exhibit such two-stage kinetics. Presenting the kinetic data in the form of semi-logarithmic plots shows a clear increase in the rate of polymerization for each of the solvent mixtures investigated, Fig. 1b. The time at which the

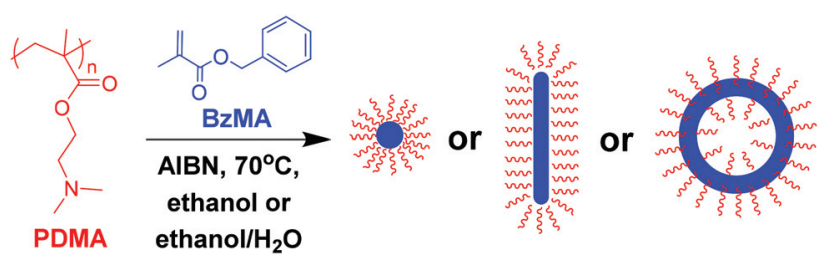

Scheme 1 Reaction scheme for the synthesis of diblock copolymer nano-objects prepared via RAFT alcoholic dispersion polymerization of benzyl methacrylate (BzMA) at $70{ }^{\circ} \mathrm{C}$ using a poly(2-(dimethylamino) ethyl methacrylate) (PDMA) chain transfer agent. AIBN initiator was used at a [PDMA]/[AIBN] molar ratio of 5 . The final diblock copolymer morphology can be spheres, worms or vesicles, depending on the precise diblock copolymer composition. 

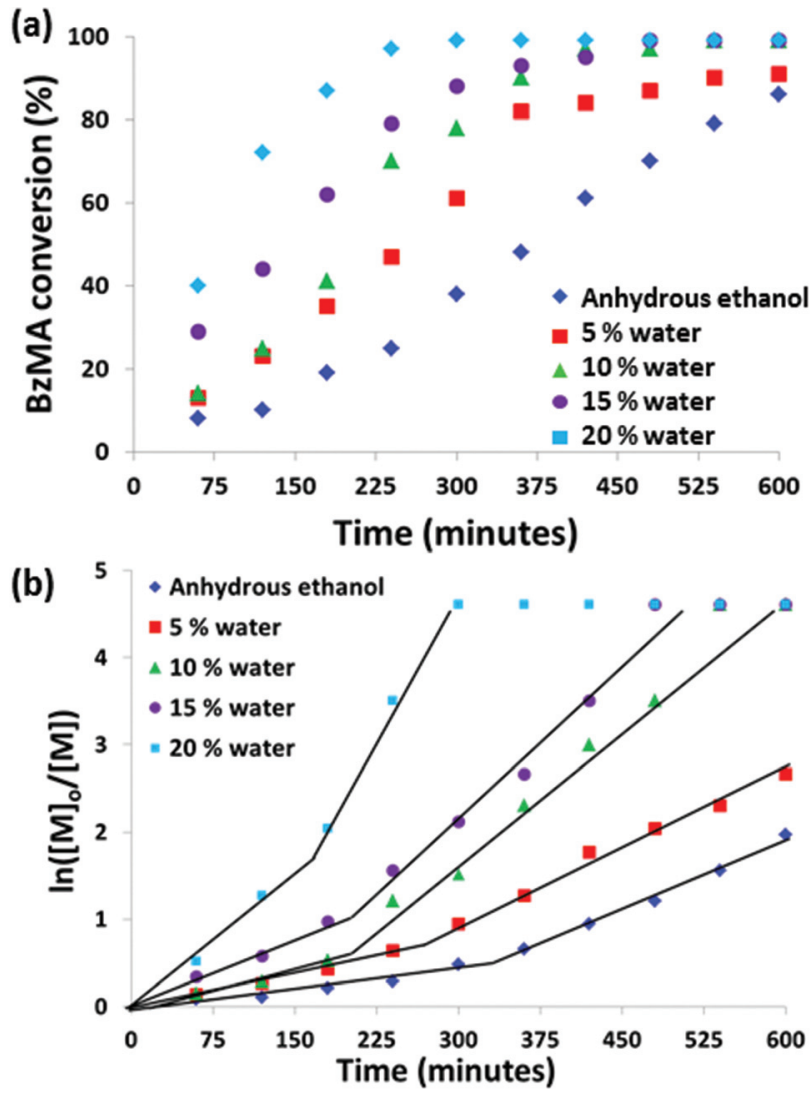

Fig. 1 Kinetic data derived from ${ }^{1} \mathrm{H}$ NMR spectroscopy studies for the RAFT dispersion polymerization of benzyl methacrylate (BzMA) at $70^{\circ} \mathrm{C}$ using a $\mathrm{PDMA}_{43}$ macro-CTA and AIBN initiator ([PDMA]/[AIBN] = 5.0) at $15 \% \mathrm{w} / \mathrm{w}$ solids. The reaction solvent was either anhydrous ethanol or various ethanol/water mixtures with a target composition of $\mathrm{PDMA}_{43}-$ $\mathrm{PBzMA}_{200}$ in each case. The same data are presented as (a) a conversion-time plot, and (b) a semi-logarithmic plot.

rate enhancement is observed corresponds to somewhat different PBzMA DPs (estimated from the conversiontime plot) depending on the solvent mixture. Surprisingly, these DPs are significantly higher than might be expected for the onset of micellar nucleation. Similar results have been recently reported by Lopez-Oliva and co-workers for PISA syntheses involving the polymerization of BzMA in $n$-heptane. ${ }^{36}$

${ }^{1} \mathrm{H}$ NMR studies in deuterated ethanol were conducted in order to examine the rate enhancement caused by partitioning of the unreacted monomer within the growing nanoparticles. Using an inert internal NMR standard (tetramethylsilane, TMS) enables conversion to be monitored by comparing the integral of the signal assigned to the internal standard (TMS, $0 \mathrm{ppm}$ ) to those of the residual monomer vinyl signals (BzMA, 5.6 and $6.1 \mathrm{ppm}$ ) and normalizing relative to the monomer integrals recorded at zero time. PBzMA is insoluble in deuterated ethanol so this block should not produce an NMR signal unless it is solvated by the unreacted BzMA monomer. A PISA synthesis targeting $\mathrm{PDMA}_{43}-\mathrm{PBzMA}_{500}$ was conducted in deut-

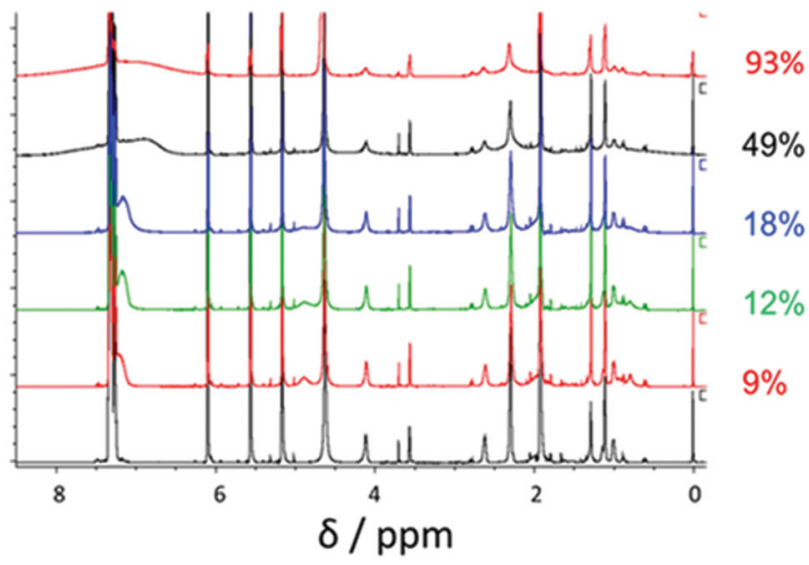

Fig. $2{ }^{1} \mathrm{H}$ NMR spectra recorded during the RAFT dispersion polymerization of BzMA at $70{ }^{\circ} \mathrm{C}$ in $\mathrm{d}_{6}$-ethanol targeting a final diblock composition of $\mathrm{PDMA}_{43}-\mathrm{PBzMA}_{500}$. Monomer conversions were calculated by comparing the integrals of the vinyl monomer to the $t=0$ spectrum using TMS as the internal standard.

erated ethanol at $70{ }^{\circ} \mathrm{C}$ at $10 \% \mathrm{w} / \mathrm{w}$ solids in a sealed NMR tube. Fig. 2 shows a series of spectra recorded at various stages of this polymerization.

As the BzMA polymerization proceeds, PBzMA signals appear at around $4.8 \mathrm{ppm}$ and $7.2 \mathrm{ppm}$. These features are clearly visible up to a monomer conversion of $18 \%$ but become weaker at higher conversions, with the $5.8 \mathrm{ppm}$ signal disappearing completely. This agrees with the hypothesis that unreacted monomer solvates the core-forming PBzMA chains. As the polymerization proceeds, there are four PDMA-PBzMA regimes to be considered, see Fig. 3. Initially, PDMA macroCTA, BzMA monomer and AIBN initiator are all dissolved in the reaction solvent. On heating, the polymerization starts and PBzMA chains begin to grow; at this stage they are sufficiently short that the PDMA-PBzMA chains remain soluble (1). Polymerization continues in homogeneous solution until the critical DP for PBzMA insolubility is attained and nucleation occurs (2). At this stage, the unreacted BzMA monomer partitions within the micelles, leading to partial solvation of the PBzMA chains. As the polymerization continues, an enhanced rate is observed (see Fig. 1) as a result of this high local BzMA concentration (3). As the PBzMA chains grow longer, the

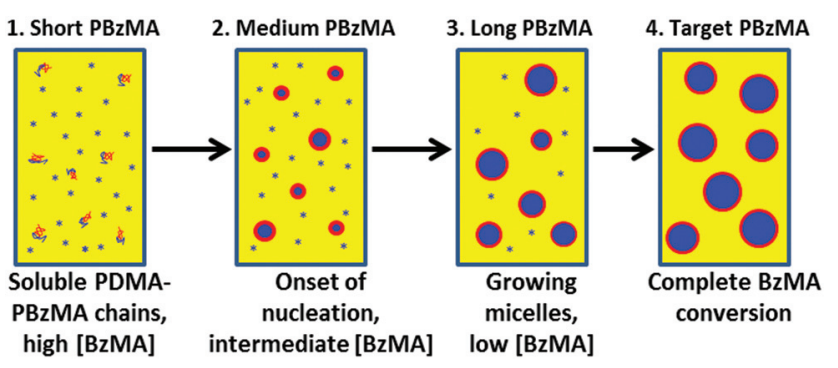

Fig. 3 Schematic cartoon illustrating the four regimes that occur during the ethanolic RAFT dispersion polymerization of BzMA. 
amount of unreacted BzMA monomer is gradually reduced, so the micelle cores are no longer sufficiently solvated to observe their characteristic aromatic and benzylic proton signals (see Fig. 2). At the end of the polymerization, almost full BzMA conversion is achieved, so no PBzMA NMR signals are expected under these conditions (4).
A similar NMR study was conducted in a mixture of deuterated ethanol and $\mathrm{D}_{2} \mathrm{O}$. Due to the faster polymerization rate under these conditions, high BzMA conversions were observed even for the early spectra (i.e. $15 \%$ after $1 \mathrm{~h}$, and $45 \%$ conversion after $2 \mathrm{~h}$ ) hence the NMR signal at $4.8 \mathrm{ppm}$ is not discernible. The signal at $7.2 \mathrm{ppm}$ is more prominent than in the

Table 1 Summary of final BzMA monomer conversions, intensity-average DLS particle diameters, GPC molecular weights $\left(M_{n}\right)$, polydispersities $\left(M_{w} / M_{n}\right)$ and TEM morphologies obtained for PDMA $43-\mathrm{PBzMA}_{x}$, or $\mathrm{PDMA}_{74}-\mathrm{PBzMA}$, diblock copolymer nanoparticles prepared at $15 \%$ w/w solids via RAFT dispersion polymerization of BzMA in either ethanol or various ethanol/water mixtures at $70{ }^{\circ} \mathrm{C}, \mathrm{PDMA} 43 / \mathrm{AIBN}$ molar ratio $=5.0$

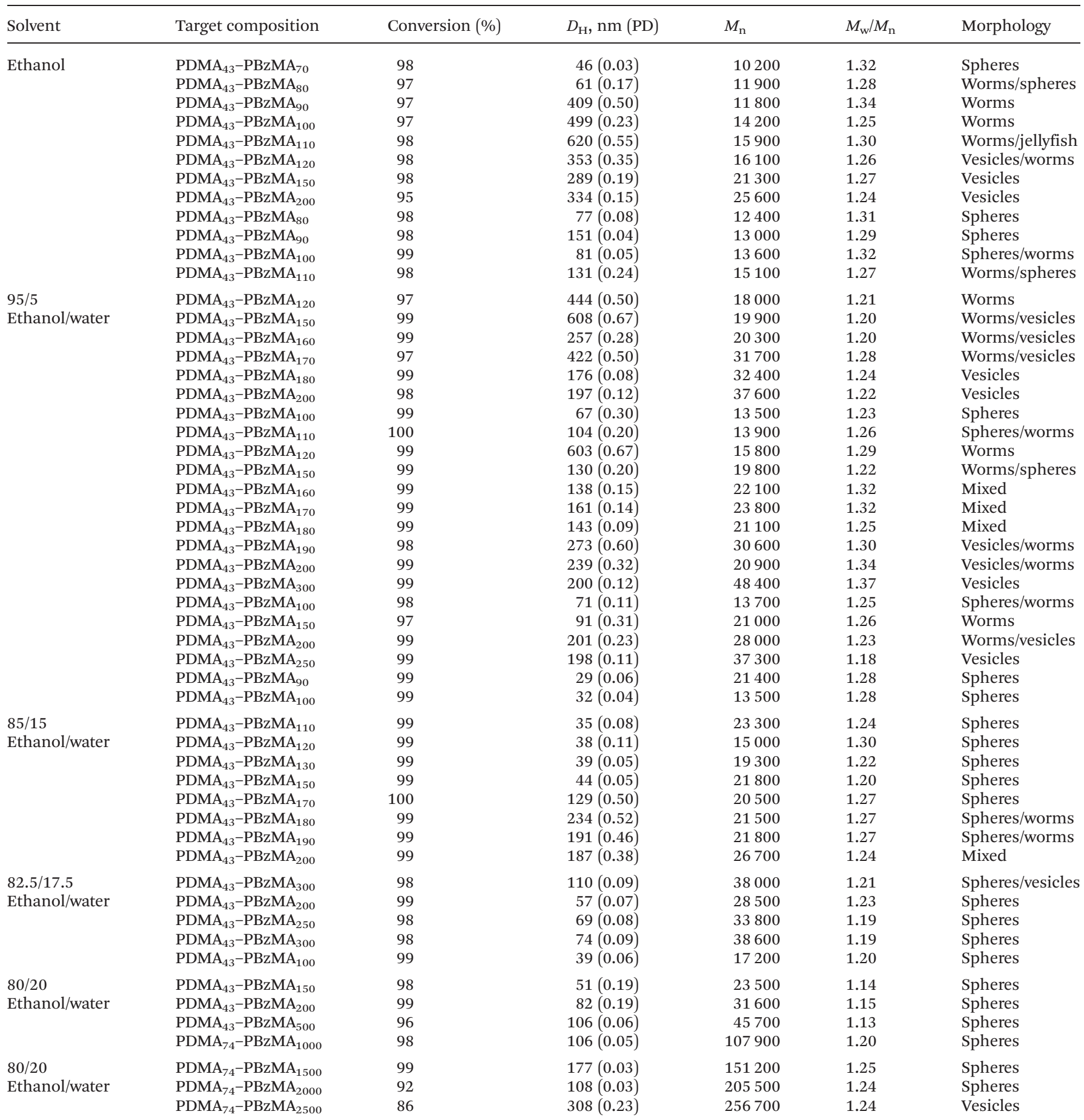


spectra for BzMA polymerization in deuterated ethanol at similar monomer conversions up to approximately $90 \%$ BzMA conversion. This provides some evidence to support the observed increase in polymerization rate on addition of water being the result of greater partitioning of BzMA monomer within micelles.

Another consideration when adding water as a co-solvent is that some of the PDMA chains may become protonated. This is because the $\mathrm{pH}$ of DI water is $\approx 6$, which is below the PDMA $\mathrm{p} K_{\mathrm{a}}$ of $7,,^{37,38}$ thus resulting in the protonated stabilizer chains acquiring cationic charge. Previous work has shown that the block copolymer self-assembly that occurs during aqueous PISA syntheses is typically limited to ill-defined spherical micelles (or in some cases prevented completely) when polyelectrolytic stabilizers are utilized. ${ }^{39,40}$ In order to see if the PISA was affected by the addition of water, a series of polymerizations were conducted targeting different PBzMA DPs using various solvent mixtures, Table 1 summarizes the results of these polymerizations. The resultant morphologies were observed by TEM, see Fig. 4, and plotted to give a phase diagram with PBzMA DP and solvent composition (as opposed to solids content) being the variables, see Fig. 5 . This phase diagram clearly demonstrates that the addition of water has a marked effect on the morphology obtained for a given diblock copolymer composition. As the water content of the continuous phase is gradually increased, lower order morphologies become more common, with only spheres being observed at $20 \% \mathrm{w} / \mathrm{w}$ water (up to a PBzMA DP of 500). The gradual change

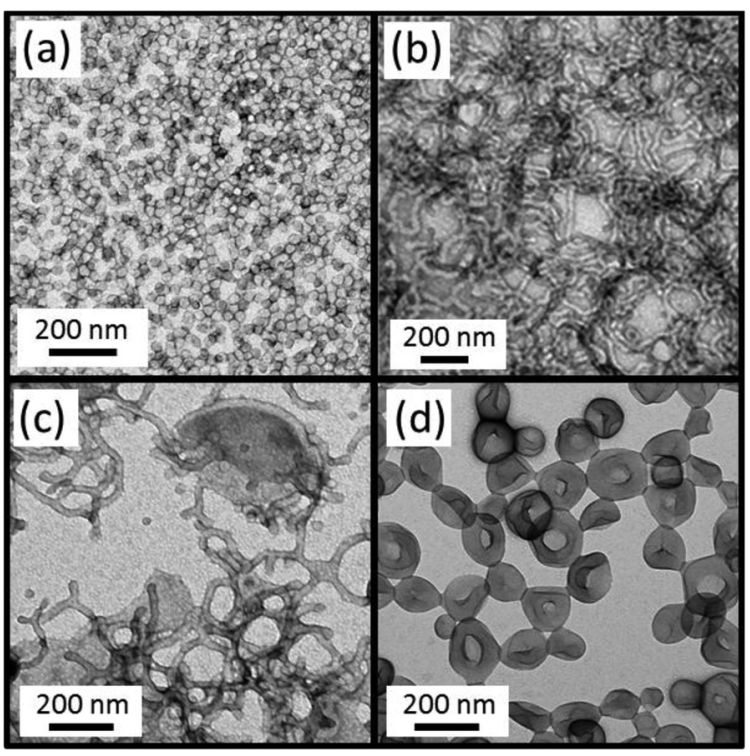

Fig. 4 Representative TEM images for $\mathrm{PDMA}_{43}-\mathrm{PBzMA}_{x}$ diblock copolymers synthesized via RAFT dispersion polymerization in anhydrous ethanol or various ethanol/water mixtures at $70^{\circ} \mathrm{C}$, with AIBN as initiator $\left(\mathrm{PDMA} / \mathrm{AlBN}\right.$ molar ratio $=5.0$ ). (a) $\mathrm{PDMA}_{43}-\mathrm{PBzMA}_{100}$ spheres synthesized in $80 / 20 \mathrm{w} / \mathrm{w}$ ethanol/water, (b) $\mathrm{PDMA}_{43}-\mathrm{PBzMA}_{100}$ worms synthesized in ethanol, (c) $\mathrm{PDMA}_{43}-\mathrm{PBz} \mathrm{MA}_{160}$ mixed worms/vesicles synthesized in 95/5 w/w ethanol/water and (d) PDMA $43-\mathrm{PBzMA}_{300}$ vesicles synthesized in $90 / 10 \mathrm{w} / \mathrm{w}$ ethanol/water.

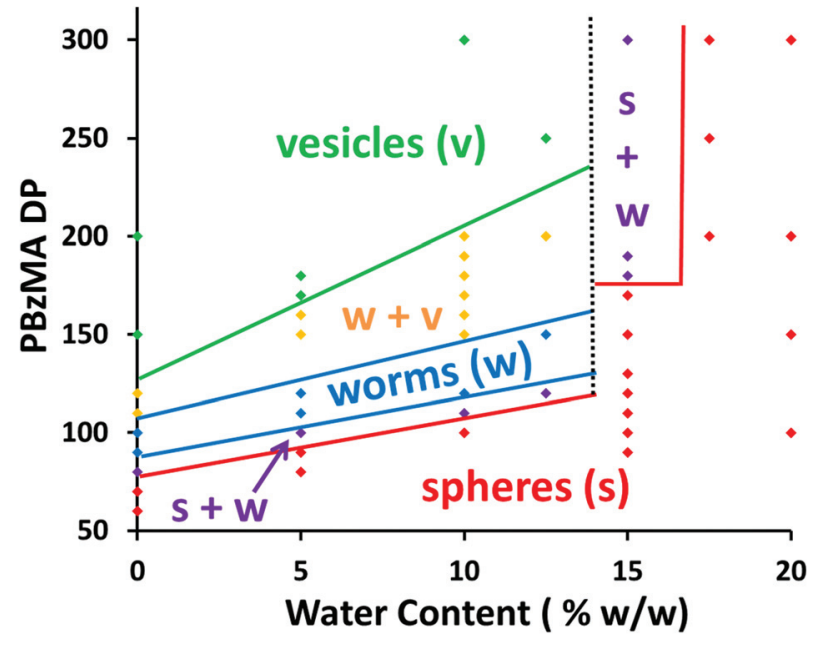

Fig. 5 Phase diagram showing the morphology obtained for $\mathrm{PDMA}_{43}-$ $\mathrm{PBzMA}_{x}$ diblock copolymers synthesized via RAFT dispersion polymerization of BzMA at $70{ }^{\circ} \mathrm{C}$ in either anhydrous ethanol or various ethanol/ water mixtures at $15 \% \mathrm{w} / \mathrm{w}$ solids. Conditions: $\left[\mathrm{PDMA}_{43}\right] /[\mathrm{AIBN}]=5.0$.

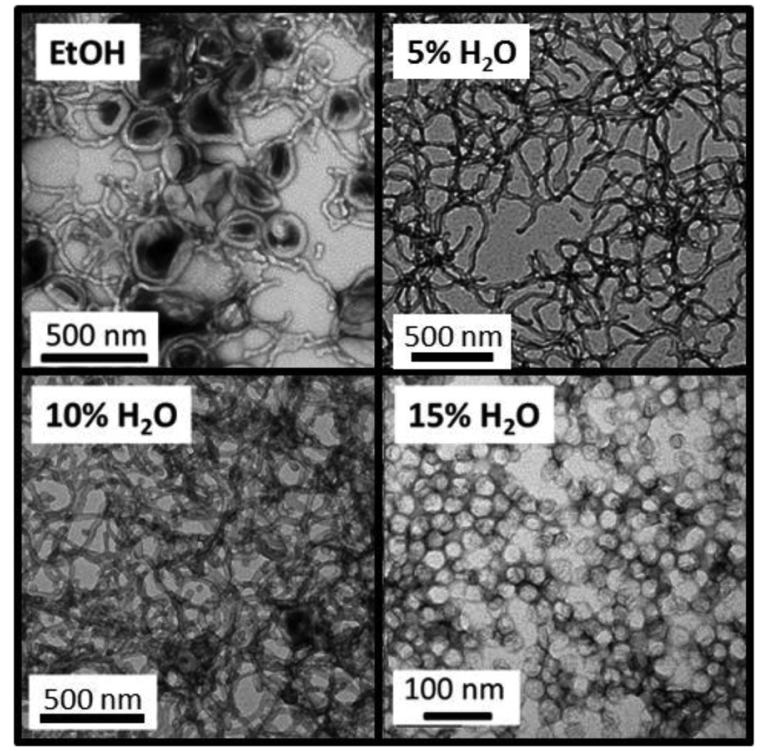

Fig. 6 TEM images of $\mathrm{PDMA}_{43}-\mathrm{PBzMA}_{120}$ nano objects prepared via RAFT dispersion polymerization of BzMA at $15 \% \mathrm{w} / \mathrm{w}$ in anhydrous ethanol or ethanol/water at $70{ }^{\circ} \mathrm{C}$ using AIBN initiator ([PDMA 43$] /[A I B N]$ $=5$ ). The solvent composition is indicated in each case and the morphology varies from a mixed vesicle/worm phase to pure worms to mixed worms/spheres and finally pure spheres as the water content of the continuous phase is increased from $0 \%$ to $15 \% \mathrm{w} / \mathrm{w}$.

in copolymer morphology for the same diblock composition is illustrated in Fig. 6. These four TEM images are for PDMA ${ }_{43}-$ PBzMA $_{120}$ diblock copolymer nanoparticles, with the difference in copolymer morphology being solely due to the varying water content of the polymerization medium.

In order to test the hypothesis of the change in morphology being due to the build-up of cationic charge on the PDMA 




Fig. 7 TEM images obtained for $\mathrm{PDMA}_{43}-\mathrm{PBzMA}_{x}$ diblock copolymer nanoparticles synthesized in either $90 / 10 \mathrm{w} / \mathrm{w}$ ethanol/water $(a, b$ and $c)$ or $90 / 10 \mathrm{w} / \mathrm{w}$ ethanol $/ \mathrm{HCl}$ ( $d$, e and f) mixtures at $15 \% \mathrm{w} / \mathrm{w}$ solids. (a) and (d) $x=100$ produced spheres in both cases. (b) and (e) $x=150$ gave a mixed phase of spheres and worms in ethanol/water whereas a pure sphere phase is obtained if the water is replaced with $\mathrm{HCl}$. (c) and (f) $x=$ 300 produces vesicles in ethanol/water but again only spheres are observed when using $\mathrm{HCl}$.

stabilizer on addition of water, reactions targeting various PBzMA DPs were conducted with $1 \mathrm{M}$ hydrochloric acid $(\mathrm{HCl})$ used in place of water. Switching the co-solvent from water to acid should result in extensive protonation of the PDMA residues, thus giving a cationic stabilizer. Fig. 7 shows the TEM images for BzMA polymerizations conducted at $15 \% \mathrm{w} / \mathrm{w}$ solids, in 90/10 w/w ethanol/water or ethanol/1 M HCl.

Comparing the morphologies of $\mathrm{PDMA}_{43}-\mathrm{PBzMA}_{x}$ synthesized in ethanol/water or ethanol/acid (see Fig. 7), only spheres are obtained up to a DP of 300 in the presence of acid. However, when synthesized in ethanol/water mixtures PDMA $_{43}-$ PBzMA $_{150}$ forms a mixed phase of spheres and worms (Fig. 7b) and $\mathrm{PDMA}_{43}-\mathrm{PBzMA}_{300}$ forms vesicles (Fig. 7c). ${ }^{1} \mathrm{H}$ NMR indicated $\geq 98 \%$ BzMA conversion for each of these polymerizations. Furthermore, THF GPC analysis of diblock copolymers with the same target composition synthesized in ethanol/water or ethanol/acid mixtures gave similar $M_{\mathrm{n}}$ values, indicating that the copolymer chains have comparable PBzMA DPs, see Table 2.
Table 2 Summary of THF GPC molecular weights $\left(M_{n}\right)$ and polydispersities $\left(M_{\mathrm{w}} / M_{\mathrm{n}}\right)$ for PDMA ${ }_{43}-\mathrm{PBzMA}_{x}$ diblock copolymers synthesized at $70{ }^{\circ} \mathrm{C}$ in either $90 / 10 \mathrm{w} / \mathrm{w}$ ethanol/water or $90 / 10 \mathrm{w} / \mathrm{w}$ ethanol $/ 1 \mathrm{M} \mathrm{HCl}$ at $15 \% \mathrm{w} / \mathrm{w}$ solids using AIBN initiator ([PDMA] $/[\mathrm{AIBN}]=5.0$ )

\begin{tabular}{|c|c|c|c|c|}
\hline \multirow{2}{*}{$\begin{array}{l}\text { Solvent } \\
\text { Composition }\end{array}$} & \multicolumn{2}{|c|}{$\begin{array}{l}\text { 90/10 w/w ethanol/ } \\
\text { water }\end{array}$} & \multicolumn{2}{|c|}{$\begin{array}{l}\text { 90/10 w/w ethanol/ } \\
1 \mathrm{M} \mathrm{HCl}\end{array}$} \\
\hline & $M_{\mathrm{n}}$ & $M_{\mathrm{w}} / M_{\mathrm{n}}$ & $M_{\mathrm{n}}$ & $M_{\mathrm{w}} / M_{\mathrm{r}}$ \\
\hline $\mathrm{PDMA}_{43}-\mathrm{PBzMA}_{100}$ & 13500 & 1.23 & 14900 & 1.32 \\
\hline $\mathrm{PDMA}_{43}-\mathrm{PBzMA}_{150}$ & 19800 & 1.32 & 21400 & 1.22 \\
\hline $\mathrm{PDMA}_{43}-\mathrm{PBzMA}_{300}$ & 48400 & 1.37 & 46200 & 1.31 \\
\hline
\end{tabular}

These results suggest that the copolymer morphology may be limited to spheres in the presence of aqueous $\mathrm{HCl}$, since the same composition gives higher order structures in the absence of acid. These observations support the hypothesis that cationic charge on the PDMA stabilizer chains leads to a significant reduction in the packing parameter, thus making spheres the preferred morphology.

In order to confirm this hypothesis, aqueous electrophoresis measurements were conducted to compare the zeta potentials of particles synthesized in ethanol/water and ethanol/hydrochloric acid mixtures. The particles synthesized in 90/10 ethanol/water have a relatively low cationic surface charge, with a zeta potential of $+7.9 \mathrm{mV}$. The particles synthesized in the presence of acid have a much higher cationic charge, giving a zeta potential of $+44.0 \mathrm{mV}$. These results provide strong evidence that charge build-up within the stabilizer layer is indeed the cause of the observed difference in morphology when the solvent is changed.

Finally, we decided to investigate whether the faster rate of polymerization observed in the presence of water co-solvent enabled higher PBzMA DPs to be achieved compared to those already reported in the literature. ${ }^{14,29,30}$ However, using the $\mathrm{PDMA}_{43}$ macro-CTA led to phase separation when targeting a DP of 1200 or higher for BzMA polymerizations conducted in $80 / 20$ ethanol/water mixtures. In view of this problem, a longer $\mathrm{PDMA}_{74}$ macro-CTA was examined, which led to colloidally stable dispersions being obtained within $24 \mathrm{~h}$ (see last four entries in Table 1). At least 98\% BzMA conversion was achieved when targeting a DP of either 1000 or 1500. Increasing the target PBzMA DP to 2000 gave 92\% BzMA conversion. For a target DP of 2500, the final monomer conversion was somewhat lower ( $86 \%$ after $24 \mathrm{~h}$ at $70{ }^{\circ} \mathrm{C}$ ). However, this still represents a marked improvement relative to that observed when closely-related PISA syntheses were conducted in pure ethanol, for which only $45 \%$ conversion was obtained when targeting a DP of 1000. TEM images were also obtained for this mini-series of $\mathrm{PDMA}_{74}-\mathrm{PBzMA}_{1000-2500}$ diblock copolymer nanoparticles, see Fig. 8. When targeting a PBzMA DP of 2000, spherical nanoparticles were obtained, but large polydisperse vesicles were produced when targeting a DP of 2500. This indicates that varying the solvent composition merely shifts the phase boundaries, as opposed 


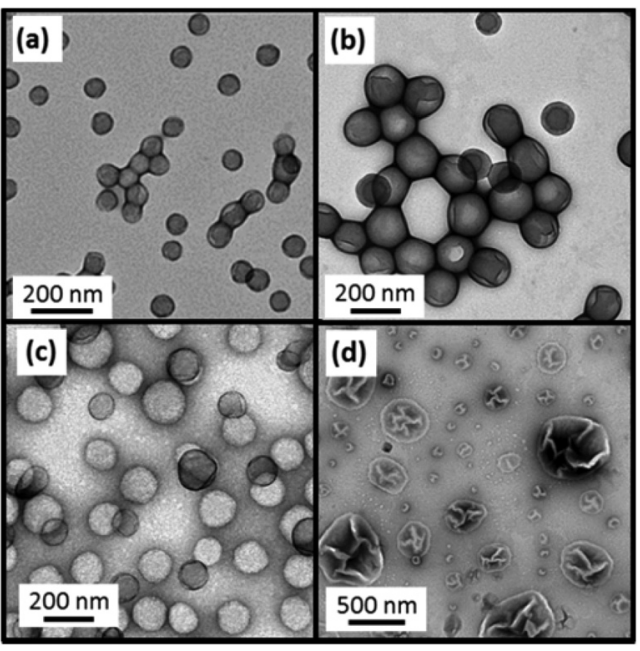

Fig. 8 TEM images showing the various copolymer morphologies obtained for $\mathrm{PDMA}_{74}-\mathrm{PBzMA}_{x}$ diblock copolymer nanoparticles synthesized in $80 / 20 \mathrm{w} / \mathrm{w}$ ethanol/water mixtures; spherical micelles for (a) $\mathrm{PDMA}_{74}-\mathrm{PBzMA}_{980}$ (98\% BzMA conversion), (b) $\mathrm{PDMA}_{74}-\mathrm{PBzMA}_{1500}$ (>99\% conversion) and (c) $\mathrm{PDMA}_{74}-\mathrm{PBzMA}_{1840}$ (92\% conversion) and vesicles for (d) $\mathrm{PDMA}_{74}-\mathrm{PBzMA}_{2150}$ (86\% conversion).

to producing kinetically-trapped spheres as the sole copolymer morphology.

\section{Conclusions}

The rate of BzMA polymerization can be substantially enhanced via addition of water co-solvent to an ethanolic RAFT dispersion polymerization formulation. This approach enables high monomer conversions to be achieved within just $6 \mathrm{~h}$ even when targeting PBzMA DPs up to 1500. This rate enhancement is attributed to greater partitioning of the BzMA monomer, which is soluble in ethanol but insoluble in water. This means that unreacted BzMA monomer preferentially enters the growing particles, increasing the local monomer concentration and thus increasing the rate of BzMA polymerization. Adjusting the solvent composition also led to different copolymer morphologies being observed for a given diblock copolymer composition. For example, $\mathrm{PDMA}_{43}-\mathrm{PBzMA}_{120}$ formed a mixed phase of vesicles plus worms when prepared in anhydrous ethanol, pure worms in the presence of $5 \% \mathrm{w} / \mathrm{w}$ water, worms plus spheres in the presence of $10 \% \mathrm{w} / \mathrm{w}$ water, but exclusively kinetically-trapped spheres in the presence of $20 \% \mathrm{w} / \mathrm{w}$ water. These observations are attributed to the PDMA stabilizer chains acquiring partial cationic charge; since this results in more efficient inter-particle repulsion, it prevents the sphere-sphere fusion events that are required for an evolution in copolymer morphology. This hypothesis was tested by replacing the water co-solvent with $1 \mathrm{M}$ aqueous $\mathrm{HCl}$. This led to the copolymer morphology being limited to spheres for all targeted diblock compositions, suggesting that the PDMA charge density strongly influences the final copolymer morphology.
In summary, the addition of water co-solvent to this alcoholic RAFT PISA formulation results in a substantially enhanced rate of BzMA polymerization. This enables the effective upper limit DP for the PBzMA core-forming block to be increased from approximately 800 for pure ethanol up to around 2000 for an $80 / 20 \mathrm{w} / \mathrm{w}$ ethanol/water mixture at $15 \%$ solids. However, it also limits access to higher order copolymer morphologies, with kinetically-trapped spheres typically being obtained in the presence of $20 \% \mathrm{w} / \mathrm{w}$ water.

\section{Acknowledgements}

DSM Advanced Surfaces (Geleen, Netherlands) is thanked for funding a CASE award for this EPSRC PhD studentship. SPA acknowledges a five-year ERC Advanced Investigator grant (PISA 320372). We also thank Stichting Innovatie Alliantie (Foundation Innovation Alliance) for additional project funding. Sue Bradshaw is thanked for her assistance with the high temperature NMR kinetic studies.

\section{References}

1 A. Rembaum, S. P. S. Yen, E. Cheong, S. Wallace, R. S. Molday, I. L. Gordon and W. J. Dreyer, Macromolecules, 1976, 9, 328-336.

2 R. Arshady, Biomaterials, 1993, 14, 5-15.

3 S. Margel, J. Chromatogr., 1989, 462, 177-189.

4 A. T. Skjeltorp, Phys. Rev. Lett., 1983, 51, 2306-2309.

5 G. Delaittre, J. Nicolas, C. Lefay, M. Save and B. Charleux, Soft Matter, 2006, 2, 223-231.

6 G. Delaittre, J. Nicolas, C. Lefay, M. Save and B. Charleux, Chem. Commun., 2005, 614-616.

7 K. H. Kim, J. Kim and W. H. Jo, Polymer, 2005, 46, 2836-2840.

8 S. Sugihara, S. P. Armes and A. L. Lewis, Angew. Chem., Int. Ed., 2010, 49, 3500-3503.

9 S. Sugihara, K. Sugihara, S. P. Armes, H. Ahmad and A. L. Lewis, Macromolecules, 2010, 43, 6321-6329.

10 J. Rieger, F. Stoffelbach, C. Bui, D. Alaimo, C. Jerome and B. Charleux, Macromolecules, 2008, 41, 4065-4068.

11 W. J. Zhang, F. D'Agosto, O. Boyron, J. Rieger and B. Charleux, Macromolecules, 2011, 44, 7584-7593.

12 Y. Li and S. P. Armes, Angew. Chem., Int. Ed., 2010, 49, 4042-4046.

13 A. Blanazs, S. P. Armes and A. J. Ryan, Macromol. Rapid Commun., 2009, 30, 267-277.

14 E. R. Jones, M. Semsarilar, A. Blanazs and S. P. Armes, Macromolecules, 2012, 45, 5091-5098.

15 L. A. Fielding, M. J. Derry, V. Ladmiral, J. Rosselgong, A. M. Rodrigues, L. P. D. Ratcliffe, S. Sugihara and S. P. Armes, Chem. Sci., 2013, 4, 2081-2087.

16 Z. An, Q. Shi, W. Tang, C.-K. Tsung, C. J. Hawker and G. D. Stucky, J. Am. Chem. Soc., 2007, 129, 14493-14499.

17 M. J. Monteiro and M. F. Cunningham, Macromolecules, 2012, 45, 4939-4957. 
18 G. Liu, Q. Qiu, W. Shen and Z. S. An, Macromolecules, 2011, 44, 5237-5245.

19 Y. W. Pei and A. B. Lowe, Polym. Chem., 2014, 5, 2342-2351.

20 W. Cai, W. Wan, C. Hong, C. Huang and C.-Y. Pan, Soft Matter, 2010, 6, 5554-5561.

21 W. M. Wan, X. L. Sun and C. Y. Pan, Macromol. Rapid Commun., 2010, 31, 399-404.

22 C.-Q. Huang and C.-Y. Pan, Polymer, 2010, 51, 5115-5121.

23 M. Semsarilar, E. R. Jones, A. Blanazs and S. P. Armes, Adv. Mater., 2012, 24, 3378-3382.

24 Y. W. Pei, N. C. Dharsana, J. A. Van Hensbergen, R. P. Burford, P. J. Roth and A. B. Lowe, Soft Matter, 2014, 10, 5787-5796.

25 C. Gonzato, M. Semsarilar, E. R. Jones, F. Li, G. J. P. Krooshof, P. Wyman, O. O. Mykhaylyk, R. Tuinier and S. P. Armes, J. Am. Chem. Soc., 2014, 136, 11100-11106.

26 M. Semsarilar, V. Ladmiral, A. Blanazs and S. P. Armes, Polym. Chem., 2014, 5, 3466-3475.

27 M. Semsarilar, N. J. W. Penfold, E. R. Jones and S. P. Armes, Polym. Chem., 2015, 6, 1751-1757.

28 W. D. He, X. L. Sun, W. M. Wan and C. Y. Pan, Macromolecules, 2011, 44, 3358-3365.

29 E. R. Jones, M. Semsarilar, P. Wyman, M. Boerakker and S. P. Armes, Macromolecules, accepted for publication.
30 X. Zhang, J. Rieger and B. Charleux, Polym. Chem., 2012, 3, 1502-1509.

31 X. Wang, J. Xu, Y. Zhang and W. Zhang, J. Polym. Sci., Part A: Polym. Chem., 2012, 50, 2452-2462.

32 F. Huo, X. H. Wang, Y. Y. Zhang, X. Zhang, J. X. Xu and W. Q. Zhang, Macromol. Chem. Phys., 2013, 214, 902-911.

33 X. Xiao, S. He, M. Dan, Y. Su, F. Huo and W. Zhang, J. Polym. Sci., Part A: Polym. Chem., 2013, 51, 3177-3190.

34 S. E. Shim, S. Oh, Y. H. Chang, M.-J. Jin and S. Choe, Polymer, 2004, 45, 4731-4739.

35 A. Blanazs, J. Madsen, G. Battaglia, A. J. Ryan and S. P. Armes, J. Am. Chem. Soc., 2011, 133, 16581-16587.

36 A. P. Lopez-Oliva, N. J. Warren, A. Rajkumar, O. O. Mykhaylyk, M. J. Derry, K. E. B. Doncom, M. J. Rymaruk and S. P. Armes, Macromolecules, 2015, 48, 3547-3555.

37 N. G. Hoogeveen, M. A. C. Stuart, G. J. Fleer, W. Frank and M. Arnold, Macromol. Chem. Phys., 1996, 197, 2553-2564.

38 V. Butun, S. P. Armes and N. C. Billingham, Polymer, 2001, 42, 5993-6008.

39 M. Semsarilar, V. Ladmiral, A. Blanazs and S. P. Armes, Langmuir, 2012, 28, 914-922.

40 M. Semsarilar, V. Ladmiral, A. Blanazs and S. P. Armes, Langmuir, 2013, 29, 7416-7424. 